\title{
A Moving Object Detection Method Based on Improved Three-frame Difference Algorithm and Edge Information
}

\author{
Liping ZHANG ${ }^{1, a}$, Zhongkai SHAO ${ }^{1,2, b}$, JianDe WU ${ }^{1,2}$, XiaoDong WANG ${ }^{1,2}$ \\ ${ }^{1}$ Faculty of Information Engineering and Automation, Kunming University of Science and \\ Technology, Kunming, 650500, China; \\ ${ }^{2}$ Engineering Research Center for Mineral Pipeline Transportation of Yunnan Province, Kunming, \\ 650500, China \\ aemail: 1162493119@qq.com, bemail: majun_km@foxmail.com
}

\begin{abstract}
Keywords: Moving Object Detection; Improvement of Three-frame Difference Algorithm; Edge Extraction, OTSU; Morphological Processing
\end{abstract}

\begin{abstract}
The moving object detection for expressways plays a significant role in intelligent transportation. A moving object detection approach of that is proposed, which combines the improved three frame difference with edge information. For obtain the marginal information of images, first the moving object's marginal information should be extracted by Sobel operator, and then compares with the results of the improved three frame difference. Finally, a completed detection area of moving object will be dealt with by the OTSU threshold segmentation algorithm and morphology. The experimental result has verified this approach can effectively improve the blank and false edge phenomenon which can be found in the traditional one. Not only that, this approach has less calculation, high real-time ability and great stability.
\end{abstract}

\section{Introduction}

Moving object detection is a hot topic of research in machine vision, and also the foundation of analyzing video image. It is widely and successfully used in the field of intelligent security, intelligent traffic and medical image analysis, but there are still some theories and application of problems to be solved, such as the motion target occlusion, shadow removal problems. Many domestic and foreign scholars have conducted a great deal of research and practice on it in recent years, many classic moving target detection algorithms have been proposed, including the optical flow segmentation method, the background difference method and the inter frame difference method, each of these methods have its advantages and disadvantages[1].The optical flow segmentation method can completely detect the moving target in the case of moving camera, but the main drawback is of high computational complexity, has low efficiency, and is not suitable for the real-time processing of the video image sequence. Background subtraction method based on the advantage of the complete moving target extraction by comparing each pixel of the current frame to the background image, but is easily affected by illumination and other external factors. The inter frame difference do difference on two or three consecutive images and make threshold segmentation on the difference image to detect moving targets. It is applied in the stationary camera, has good real-time performance and small amount of calculation, and can better adapt to the dynamic environment, but if the pixel has no obvious change, the traditional frame difference algorithm is difficult to detect the overlap of two frames, then the result forms the "hollow" phenomenon.

HuaYuanlei introduced the 8 neighborhood sum operation into two frame difference method in the literature [2] and certainly improved the "empty" phenomenon. Typically, image edge information is less affected by the brightness of the mutation and noise [3]. Gan Minggang [5] combined the edge detection and three-frame difference to enhance robustness of the detection algorithm. In order to realize the real-time detection of moving targets in the expressway, this paper presents a new target detection method which combines the three-frame difference with edge detection. In this paper, the method effectively improves the 'empty' phenomenon caused by the 
traditional three-frame difference algorithm and reduces the influence of the noise and brightness mutation by extracting the image edge and doing 8 neighborhood summations operation on the difference image. The algorithm is of low computational complexity, has good real-time performance and robustness, and can detect the moving targets accurately.

\section{The Principle of Difference Algorithm}

Setting $f_{k-1}(x, y), f_{k}(x, y)$ are two adjacent frame images in a video sequence, $g(x, y)$ can be obtained after difference operation

$$
g(x, y)=\left|f_{k-1}(x, y)-f_{k}(x, y)\right|
$$

When the moving target which has no significant moving, the changing region which moving object covers is minimal, so it's not easy to detect the moving targets. So usually choose 3 frame images to carry out difference and operation in practical application, and achieve total dynamic trajectory accumulated the motion orbit [5,6], the method is called three-frame difference. Setting $f_{k-1}(x, y), f_{k}(x, y), f_{k+1}(x, y)$ are the three successive images in the video $g_{1}(x, y)$ and $g_{2}(x, y)$ are difference images of the first two frames and the last two frames. The image after processed by three- frame difference method named as $D(x, y)$.

$$
\begin{gathered}
g_{1}(x, y)=\left|f_{k-1}(x, y)-f_{k}(x, y)\right| \\
g_{2}(x, y)=\left|f_{k}(x, y)-f_{k+1}(x, y)\right| \\
D(x, y)=g_{1}(x, y) \otimes g_{2}(x, y)
\end{gathered}
$$

Select a certain thresholdTand do binary processing $D(x, y)$ to obtain binary image $B(x, y)$.

$$
B(x, y)=\left\{\begin{array}{c}
255, \quad D(x, y) \geq T \\
0, D(x, y)<T
\end{array}\right.
$$

The white area is the target area.

\section{Moving Target Detection Method Based on Improved Three Frame Difference}

\section{A. The Basic Flow of Algorithm}

In this paper, the improved three-frame difference is applied in moving target detection, and the algorithm of the whole process is shown in Figure 1. The method firstly uses the Sobel operator to extract the edge of three consecutive frames, secondly do the difference operation on the first two frames and the last two frames, and do the 8 neighborhood summation operation on the gotten difference images, finally accomplish the extraction of moving objects though the OTSU threshold segmentation algorithm and morphological image processing.

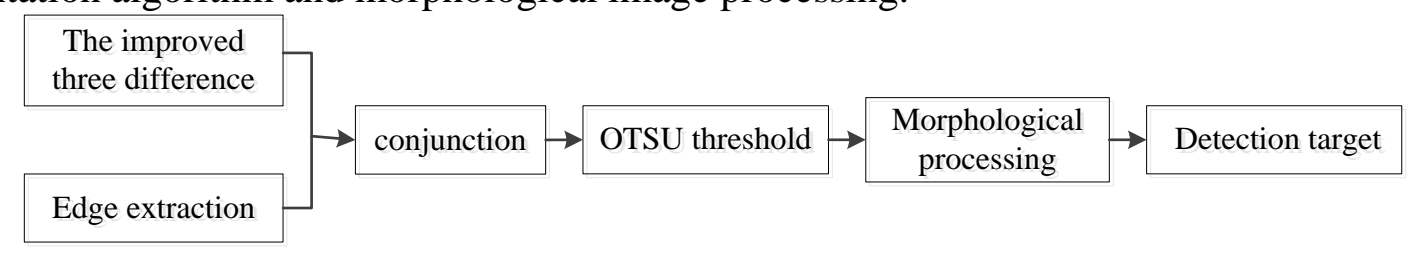

\section{B. The Edge Extraction}

Fig.1 The flow chart of the algorithm

Usually using Sobel operator extracts the image edge information. The Sobel operator combines with Gauss smoothing and differentiation, both vertically and horizontally weights the adjacent pixel values of the gray image, and has better effect of edge detection and a certain robustness to noise [6]. Comparing with other operators, such as Roberts, Prewitt, LOG and Canny operator edge extraction, the Sobel operator has good effect, small computational complexity, easy for hardware implementation and better real-time performance advantages [7-9]. The Sobel operator make the same size area in the selected window separately convolve with vertical and horizontal templates, so the image gradient value can be obtained. Gradient value that is greater than the threshold value of the part is the edge of the image. The horizontal and vertical edges can be extracted through $X$ 


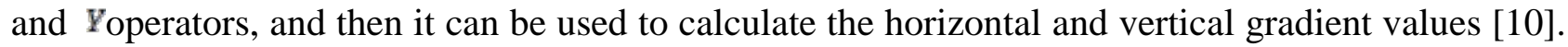
Define $f(x, y)$ as the image gray value, $H_{x}$ and $H_{y}$ separately represent $X$ and $Y_{\text {Ooperators. }}$

$$
S(x, y)=\left|f(x, y) * H_{x}\right|+\left|f(x, y)^{*} H_{y}\right|
$$

$T$ is the set threshold, when $S(x, y)>T, S(x, y)$ is the step like edge point, $\{S(x, y)\}$ is the edge images.

\section{The Improved Three frame Difference Algorithm}

Aiming at the problem of the incomplete target detection and the "empty" phenomenon, this paper proposed an improved three-frame difference algorithm which introduces the 8 neighborhood summation. The principle is as follows.

$$
g(x, y)=\left|f_{k-1}(x, y)-f_{k}(x, y)\right|
$$

In formula (6), $f_{k-1}(x, y)$ is the gray value of the previous frame, $f_{k}(x, y)$ is the gray value of the current frame.

Do the 8 neighborhood summation operation on the gotten difference images, as in table 1 below.

Table1. The 8 neighborhood of $g(x, y)$

\begin{tabular}{|c|c|c|}
\hline$g(x-1, y-1)$ & $g(x, y-1)$ & $g(x+1, y-1)$ \\
\hline$g(x-1, y)$ & $g(x, y)$ & $g(x+1, y)$ \\
\hline$g(x-1, y+1)$ & $g(x, y+1)$ & $g(x+1, y+1)$ \\
\hline
\end{tabular}

The 8 neighborhood summation process of $g(x, y)$ is as follows.

$\sum_{i} G(x, y)=g(x-1, y-1)+g(x, y-1)+g(x+1, y-1)+g(x-1, y)+g(x, y)$

$+g(x+1, y)+g(x-1, y+1)+g(x, y+1)+g(x+1, y+1)$

Conduct the 8 neighborhood summation operation on $g_{1}(x, y)$ and $g_{2}(x, y)$ to get $\sum_{i} G_{1}(x, y)$ and $\sum_{i} G_{2}(x, y)$

The improved three frames difference calculation formula is as follows.

$D_{1}(x, y)=\sum_{i} G_{1}(x, y) \otimes \sum_{i} G_{2}(x, y)$

Moving target can be detected after the threshold processing to $D_{1}(x, y)$.

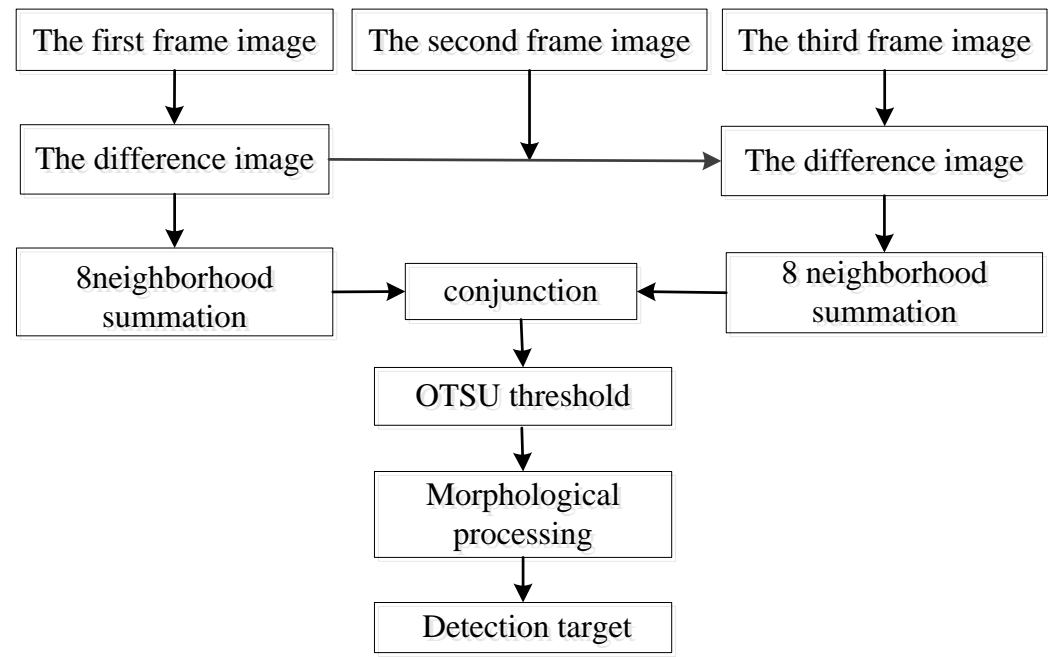

Fig. 2 The flow chart of the three-frame difference algorithm

\section{Determine Threshold Value by OTSU}

The threshold is determined by the OTSU algorithm which is based on the separation of the target and background in the image. The image histogram is divided into foreground and 
background by the best threshold value, the classification performance categories which is represented by $\lambda(t)$ depends on the selected threshold value. The best threshold value is the maximum threshold that can reach the maximum variance between foreground and background. The method can automatically select the image segmentation threshold and get the expected result.

Assume an image with $\mathrm{L}$ gray level is divided into two areas named $D_{1}$ and $D_{2}$ by threshold value $t$, the pixel value $i$ is represented by $p_{i}$.

$$
P_{D_{1}}=\sum_{i=0}^{t-1} p_{i}, P_{D_{2}}=\sum_{i=t}^{L-1} p_{i}
$$

In formula (12), the proportion of $D_{1}, D_{2}$ pixel regions in the total pixel are represented by $P_{D_{1}}$ and $P_{D_{2}}$.

$$
u=\sum_{i=0}^{L-1} i p_{i}, u_{1}=\frac{\sum_{i=0}^{t-1} i p_{i}}{P_{D_{1}}}, u_{2}=\frac{\sum_{i=t}^{L} i p_{i}}{P_{D_{2}}}
$$

In formula (13), the grayscale average of the whole pair image is represented by $u$, the grayscale average of the region $D_{1}$ is represented by $u_{1}$, and the grayscale average of the region $D_{2}$ is represented by $\boldsymbol{u}_{2}$.

$$
\begin{aligned}
& \delta_{\text {in }}=P_{D_{1}}\left[\sum_{i=0}^{t-1} p_{i}\left(i-u_{1}\right)^{2}\right]+P_{D_{2}}\left[\sum_{i=t}^{L} p_{i}\left(i-u_{2}\right)^{2}\right] \\
& \delta_{\text {out }}=P_{D_{1}}\left(i-u_{1}\right)^{2}+P_{D_{2}}\left(i-u_{2}\right)^{2}
\end{aligned}
$$

In formula (14) and (15), inter - class variance and between-class variance are represented by $\delta_{\text {in }}$ and $\delta_{\text {out }}$.

Introduce equivalent criterion associated with $t$.

$$
\lambda(t)=\frac{\delta_{\text {out }}}{\delta_{\text {in }}}
$$

When $\lambda(t)$ is at its maximum, $t$ is the best threshold.

\section{E. Morphological Processing}

Due to the changes of environmental factors and the existing noise, the binary images often have some isolated noise points, so in order to extract better and more complete moving objects, the binary images are needed for morphological filtering. Morphological processing can effectively filter out the isolated regions in the binary image and fill the small gap regions. In order to analyze and identify the image, it is the key to use unit structure with a certain shape to measure and extract the corresponding form in an image ${ }^{[11]}$. Mathematical morphology is mainly divided into erosion and dilation.

For collection $A$ and $B$, erosion operation can be defined as.

$A \Theta B=\{x: B+x \subset A\}$

Dilation operation can be defined as.

$$
A \oplus B=\left[A^{c} \Theta(-B)\right]^{c}
$$

The practical application often combined corrosion and expansion together to conduct morphological processing, which are known as the opening and closing operation.

The opening operation can be defined as the fallow.

$A \circ B=(A \Theta B) \oplus B$

The closing operation is the dual operation of the opening operation, which is defined as follows.

$A \bullet B=[A \oplus(-B)] \Theta(-B)$

The opening and closing operation can eliminate some unit structure of small image details in the 
case of the overall geometric distortion doesn't occur ${ }^{[12]}$. The opening operation can be considered to get rid of the small isolated areas and cut the slender lap, which plays a certain role in separation. The closing operation can fill the small gap on the foreground region and connect the short lap, which plays a certain role in communication. The moving target can be detected completely with cooperation of the opening and closing operation to cooperate with each other.

\section{The Experimental Results Analysis}

The paper uses the experiment to validate the detection algorithm under the environment of VS2010 and OpenCV2.4.6.The video data in the experiment is a highway vehicle video. The improving three frame difference algorithm introduces the edge feature extraction and 8 neighborhood summations to extract the moving target, which has overcome disadvantages of the two frame difference. The experimental results are shown in figure 4 . The figure $4 \mathrm{~b}$ is the detection result of the improving frame difference algorithm, figure $4 \mathrm{e}$ is the experimental result of the improving three frame difference algorithm proposed in this paper.
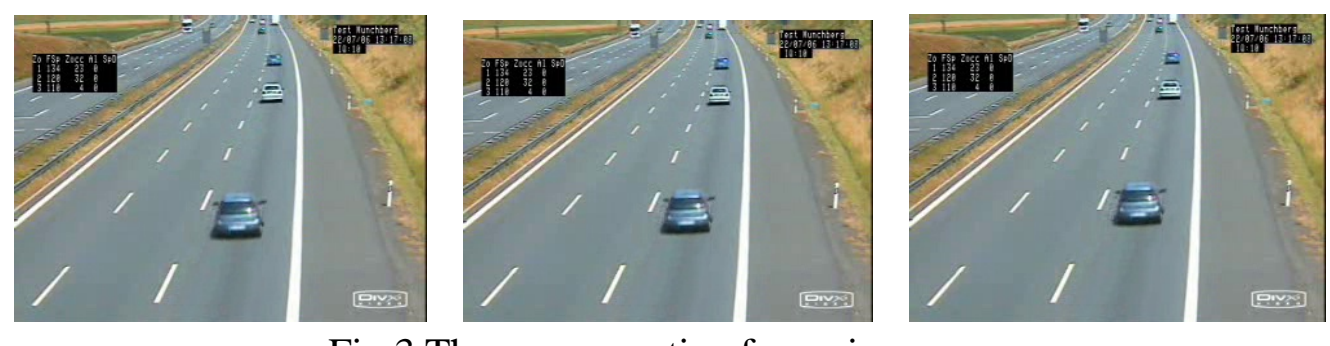

Fig.3 Three consecutive frame images

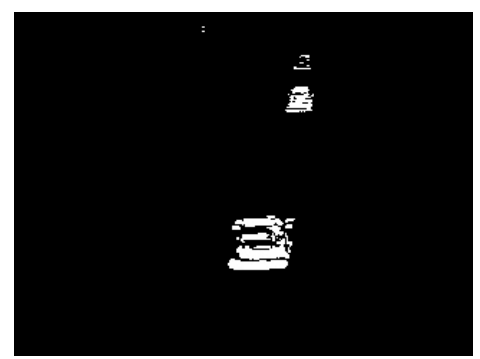

a) The traditional two frame

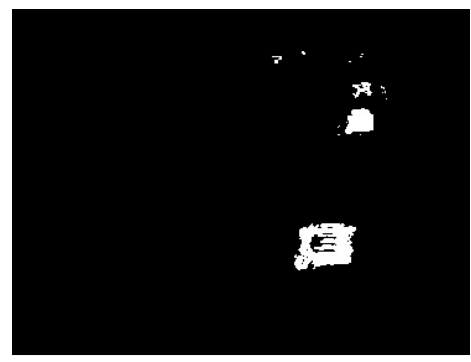

c) The traditional three- frame

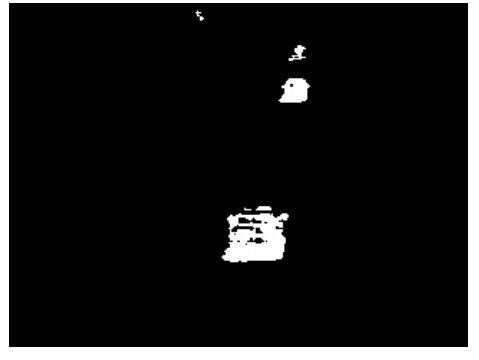

d) The improved three- frame

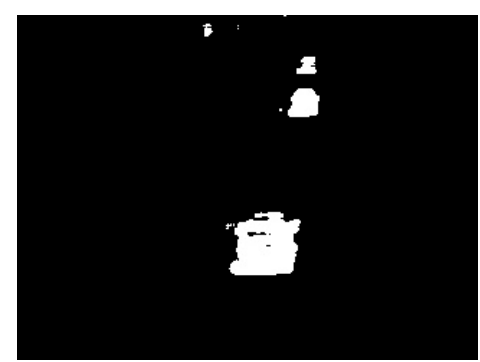

b) The improved two frame difference

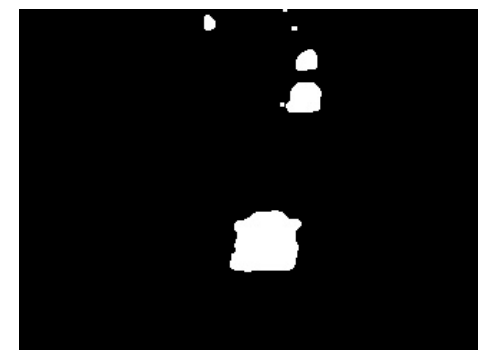

e) The final results

Fig4. The experimental results comparison

\section{Conclusions}

Through the comparative analysis of the experimental results, some useful conclusions can be obtained.

1. Doing the 8 neighborhood summations operation on the difference image has certain improved the "empty" phenomenon, but it cannot completely extract the moving target.

2. The improved three frame difference algorithm overcomes the 'empty' phenomenon happened in the moving object region caused by the two frame difference algorithm and completely extract the moving object. 
3. The improved three frame difference algorithm has strong robustness in the case of luminance mutation and completely extract the moving object. The algorithm is of low computational complexity, has good real-time performance and meet real-time requirement of highway.

But this method cannot solve shadow and occlusion problems of the moving target, which will be one of the directions to study in the future.

\section{Acknowledgement}

This work is supported by National Natural Science Foundation of China (No. 51169007), Science \& Research Program of Yunnan province (No.2011CI017 \& 2012CA022\&2013DH034).

\section{References}

[1] L.K.Qiu, Q.L.Liu, W.L.Lei. The motion detection based on background subtraction and three frame difference[J]. Journal of HeFei University of Technology, Vol.5, No.37, 572-577, 2014.

[2] Y.L.Hua, W.J.Liu. Moving target detection algorithm based on improved mixed Gauss model[J]. Computer application, Vol.34, No.2, 580-584, 2014.

[3] Tong Nian-nian and Duan Xiao-hui. Research on detection algorithm for vehicle monitoring system[C]. The Fist Chinese Conference on Intelligent Surveillance, Beijing, 2002: 612-616.

[4] R.Z.Liu, S.Q.Yu. OpenCV basic tutorial article[M]. Beijing, 612-616, 2002.

[5] L.Mo, P.Liao, X.Liu. A moving target detection algorithm based on background subtraction and three- frame difference[J]. Micro computer information, Vol.25, No.4-3, 274-276, 2009.

[6] M.G. Gan, J.Chen, J.Liu,etc. A moving target detection based on three-frame difference and edge information[J]. Journal of Electronics \& Information Technology, Vol.32, No.4, 894-897, 2010.

[7] Zhao Ming-ying and Zhao Jun. A novel method for moving object detection in intelligent video surveillance systems[C]. International Conference on Computational Intelligence and Security, Guangzhou, 2006, 2: 1797-1800.

[8] Xie Xiao-zhu, Hong Jing-xin, and Xie Si-xing. Effective method for moving objects detection on sea surface[C]. International Conference on Computer Science and Software Engineering, Wuhan, 2008, 3: 1-4.

[9] Jin Peng-fei. Improved algorithm for sobel edge detection of image[J]. Journal of Applied Optics, 2008, 29(4): 625-628.

[10] M.C.Chai. The complete extraction scheme of Edge detection [J].The brand, No.9, 169-169, 2014.

[11] J.S.Zheng. The reach on extraction algorithm for vehicle edge based on adaptive threshold[J]. Journal of Shandong Jiaotong University, Vol.16, No.1, 23-25, 2008.

[12] P.Tian, J.Jin, L.Li,ect. A kind of biological characteristic recognition based on between-class variance and morphology. Journal of Tsinghua University (natural science), Vol.47, No.z2, 1747-1750, 2007. 